\title{
g
}

\section{Observation of Pair Condensation in the Quasi-2D BEC-BCS Crossover}

\author{
M. G. Ries, ${ }^{1,}$ A. N. Wenz, ${ }^{1}$ G. Zürn, ${ }^{1}$ L. Bayha,${ }^{1}$ I. Boettcher, ${ }^{2}$ D. Kedar, ${ }^{1}$ P. A. Murthy, ${ }^{1}$ \\ M. Neidig, ${ }^{1}$ T. Lompe, ${ }^{1, \dagger}$ and S. Jochim ${ }^{1}$ \\ ${ }^{1}$ Physikalisches Institut, Ruprecht-Karls-Universität Heidelberg, 69120 Heidelberg, Germany \\ ${ }^{2}$ Institut für Theoretische Physik, Ruprecht-Karls-Universität Heidelberg, 69120 Heidelberg, Germany
}

(Received 19 March 2015; published 8 June 2015)

\begin{abstract}
The condensation of fermion pairs lies at the heart of superfluidity. However, for strongly correlated systems with reduced dimensionality the mechanisms of pairing and condensation are still not fully understood. In our experiment we use ultracold atoms as a generic model system to study the phase transition from a normal to a condensed phase in a strongly interacting quasi-two-dimensional Fermi gas. Using a novel method, we obtain the in situ pair momentum distribution of the strongly interacting system and observe the emergence of a low-momentum condensate at low temperatures. By tuning temperature and interaction strength, we map out the phase diagram of the quasi-2D BEC-BCS crossover.
\end{abstract}

DOI: 10.1103/PhysRevLett.114.230401

PACS numbers: 03.75.Ss, 03.75.Hh, 67.10.Db, 67.85.Lm

The characteristics of quantum many-body systems are strongly affected by their dimensionality and the strength of interparticle correlations. In particular, strongly correlated two-dimensional fermionic systems have been of interest because of their connection to high- $T_{c}$ superconductivity. Although they have been the subject of intense theoretical studies [1-8], a complete theoretical framework has not yet been established.

Ultracold quantum gases are an ideal realization for exploring strongly interacting 2D Fermi gases, as they offer the possibility of independently tuning the dimensionality and the strength of interparticle interactions. Reducing the dimensionality [9] led to the observation of a BerezinskiiKosterlitz-Thouless (BKT)-type phase transition to a superfluid phase in weakly interacting 2D Bose gases [10,11]. Tuning the strength of interactions in a three-dimensional two-component Fermi gas made it possible to explore the crossover between a molecular Bose-Einstein Condensate (BEC) and a BCS superfluid [12-15].

Recently, efforts have been made to combine reduced dimensionality with the tunability of interactions and to experimentally explore ultracold 2D Fermi gases [16-21]. However, the phase transition to a condensed phase has not yet been observed. Here, we report on the condensation of pairs of fermions in the quasi-2D BEC-BCS crossover.

The BEC-BCS crossover smoothly links a bosonic superfluid of tightly bound diatomic molecules to a fermionic superfluid of Cooper pairs in 2D as well as $3 \mathrm{D}$ systems. However, changing the dimensionality leads to some inherent differences. In two dimensions, there is a two-body bound state for all values of the interparticle interaction. Furthermore, because of the enhanced role of fluctuations in $2 \mathrm{D}$, true long-range order is forbidden for homogeneous systems at finite temperature $[22,23]$. Still, a low temperature superfluid phase with quasi-long-range order can emerge due to the BKT mechanism $[24,25]$.
In a $2 \mathrm{D}$ gas with contact interactions, the interactions can be described by the 2D scattering length $a_{2 D}$. Using the Fermi wave vector $k_{F}$, the dimensionless crossover parameter is given by $\ln \left(k_{F} a_{2 D}\right)$. The crossover regime is reached for $\left|\ln \left(k_{F} a_{2 D}\right)\right| \lesssim 1$. For $\ln \left(k_{F} a_{2 D}\right) \ll-1$, the binding energy is large and the system consists of deeply bound bosonic dimers. For $\ln \left(k_{F} a_{2 D}\right) \gg 1$, the dimer binding energy tends to zero. For a thermal energy $k_{B} T$ significantly larger than the binding energy, the dimers are dissociated due to thermal excitations and the system becomes fermionic.

Two-dimensional gases are realized by a strongly anisotropic confinement, which leads to a freezing out of the degrees of freedom in one spatial direction. Such a quasi2D gas captures the essential properties of a 2D system. Corrections to the $2 \mathrm{D}$ physics may arise from the residual influence of the third dimension.

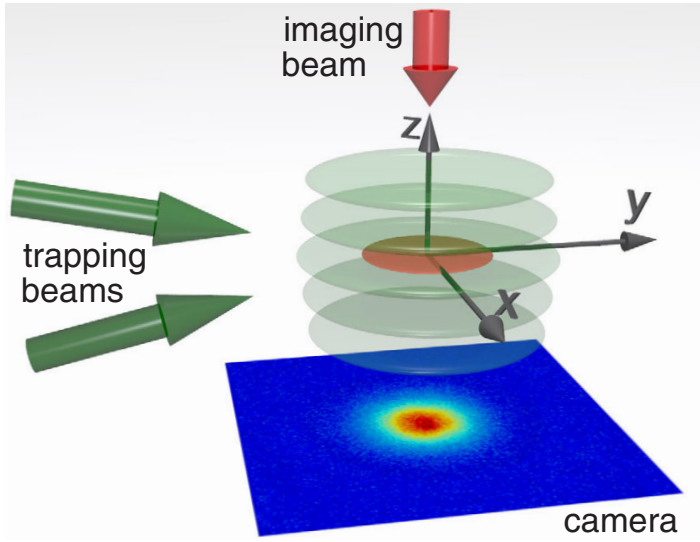

FIG. 1 (color online). Experimental setup. A quasi-2D gas (the red disk) is created by loading a two-component ultracold Fermi gas of ${ }^{6} \mathrm{Li}$ atoms into a single layer of a standing-wave trap created by two interfering laser beams $(\lambda=1064 \mathrm{~nm}$, the green arrows) that cross under a small angle $\left(14^{\circ}\right)$. Using absorption imaging along the vertical direction (the red arrow) we obtain the column density of the sample. 
We perform our measurements using a two-component Fermi gas of ${ }^{6} \mathrm{Li}$ atoms in the lowest two Zeeman sublevels, which we denote as $|1\rangle$ and $|2\rangle$ [26]. The ultracold gas initially consists of 40000-50000 atoms per spin state, which are bound into dimers at a temperature of approximately $50 \mathrm{nK}$ and a magnetic offset field of $795 \mathrm{G}$ $\left(\ell_{z} / a_{3 D}=1.08\right)$ [27]. It is loaded into a hybrid trap consisting of a single layer of a standing-wave optical dipole potential and a weak magnetic potential. The combined trapping frequencies are $\omega_{x}=2 \pi \times 17.88(3) \mathrm{Hz}$ and $\omega_{y}=2 \pi \times 17.82(4) \mathrm{Hz}$ in radial, and $\omega_{z}=$ $2 \pi \times 5.53(3) \mathrm{kHz}$ in axial direction. This leads to a pancake-shaped cloud with an aspect ratio of $\omega_{z} / \omega_{r} \approx 310$ (see Fig. 1) and an axial harmonic oscillator length $\ell_{z}=$ $\sqrt{\hbar / m \omega_{z}} \approx 551 \mathrm{~nm}$ with the reduced Planck's constant $\hbar$, the atom mass $m$, and the axial trapping frequency $\omega_{z}$. We ensure that there is no significant population of axially excited states by measuring the axial momentum distribution of the gas $[18,27]$. Assuming that the internal structure of pairs, i.e., the relative wave function of the fermions inside the pairs, has only negligible effect beyond the two-body sector [36], our system can be described in the $2 \mathrm{D}$ framework with the effective $2 \mathrm{D}$ scattering length $a_{2 D}=\ell_{z} \sqrt{\pi / A} \exp \left[-\sqrt{\pi / 2}\left(\ell_{z} / a_{3 D}\right)\right][3,16,27,37]$, where $A=0.905$.

To explore the phase diagram of the quasi-2D BEC-BCS crossover, we tune the temperature by heating the sample, and the interaction strength by adiabatically ramping the magnetic offset field to values between $692 \mathrm{G}$ $\left(\ell_{z} / a_{3 D}=7.11\right)$ and $982 \mathrm{G}\left(\ell_{z} / a_{3 D}=-2.35\right)$ [27]. We probe the $2 \mathrm{D}$ density distribution via absorption imaging along the vertical direction (see Fig. 1). The density distributions for different interaction strengths are shown in Fig. 2(a) for the coldest accessible temperatures. For growing $\ln \left(k_{F} a_{2 D}\right)$, the width of the sample increases while its central density decreases from approximately $2.7 / \mu \mathrm{m}^{2}$ at $\ln \left(k_{F} a_{2 D}\right)=-7.13$ to approximately $0.76 / \mu \mathrm{m}^{2}$ at $\ln \left(k_{F} a_{2 D}\right)=3.24$. This change of the density distribution illustrates the crossover from a dense condensate of bosonic molecules to a degenerate Fermi gas whose density is reduced by the Fermi pressure. However the phase transition into a condensed phase, which manifests itself in the enhanced density of pairs with vanishing momentum, is not directly visible in the measured density distributions.

We thus conceived a method to probe the in situ pair momentum distribution of our strongly interacting system by combining a quench of interactions with a matter wave focusing technique in which the sample expands ballistically in a weakly confining radial harmonic potential [38-41].

Because of its large aspect ratio, our sample expands rapidly and almost exclusively in the $z$ direction after the release from the optical trap. Hence, its density suddenly drops and interactions between the expanding particles are quenched. Redistribution of momentum in the radial direction during the expansion is thus negligible at the weakest probed interaction strengths and does not affect the momentum distribution. To minimize interaction effects also in the strongly interacting regime, we perform a fast ramp to the lowest accessible interaction strength on the BEC side $\left(B=692 \mathrm{G}, \ell_{z} / a_{3 D}=7.11\right)$ on a time scale shorter than $125 \mu \mathrm{s}$ just before the release. This is fast enough that the density and momentum distributions cannot adjust to the new interaction parameter [27,41]. At the same time, pairs of atoms are projected onto deeply bound molecules whose binding energy $E_{B}$ significantly exceeds the energy scale given by the axial confinement $\left(\hbar \omega_{z}\right)$ and one obtains the pair momentum distribution [42]. A similar technique was already used to explore the threedimensional BEC-BCS crossover $[13,14,44]$. However, these experiments could not take advantage of the interaction quench and the subsequent ballistic expansion since they were lacking the fast expansion in the $z$ direction.

To obtain the radial momentum distribution, we perform this ballistic expansion in a weakly confining harmonic potential with trap frequency $\omega_{\exp }=2 \pi \nu_{\exp }$ in the radial direction. In a simple picture, the harmonic potential acts as a matter wave lens and brings the far field distribution to finite

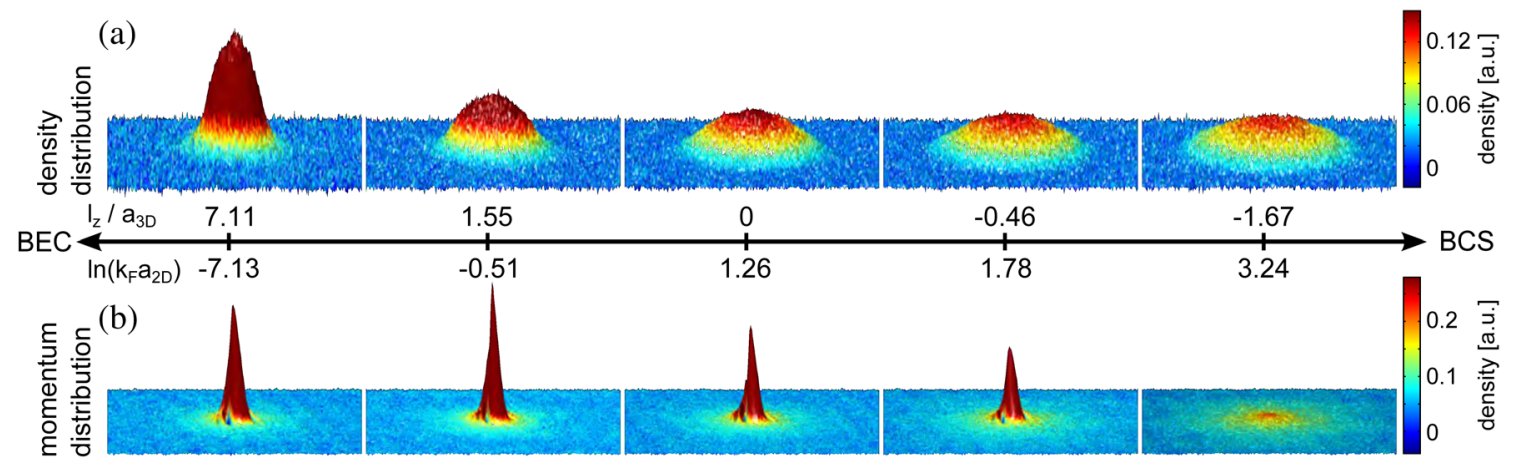

FIG. 2 (color online). Density distributions at the lowest accessible temperature for different interaction strengths. (a) In situ density distribution obtained from absorption imaging along the $z$ axis. (b) Pair momentum distribution obtained from the $\tau / 4$ method with a pair projection ramp to $\ell_{z} / a_{3 D}=7.11(692 \mathrm{G})$. The strong enhancement at low momenta in the momentum distribution for $\ln \left(k_{F} a_{2 D}\right)<3.24$ is a clear signature of pair condensation. Each picture is the average of about 30 individual measurements. The temperature of the samples ranges from $64 \mathrm{nK}$ at $\ln \left(k_{F} a_{2 D}\right)=-7.13$ to $78 \mathrm{nK}$ at $\ln \left(k_{F} a_{2 D}\right)=3.24$. 
time scales. After an expansion time of $t_{\exp }=\tau / 4$, where $\tau=1 / \nu_{\exp }$ is the period of the harmonic potential, the position of each particle depends only on its initial momentum in the radial plane. Thus $n(\mathbf{x}, t=\tau / 4)=$ $\tilde{n}\left(\hbar \mathbf{k} /\left(m \omega_{\exp }\right), t=0\right)$ and hence, by imaging the density profile after $t_{\exp }=\tau / 4$, we gain direct access to the initial $2 \mathrm{D}$ momentum distribution $[38,39,41]$. In our case, the radial trap frequency is $\omega_{\exp } \simeq 2 \pi \times 10 \mathrm{~Hz}$, which leads to $t_{\exp }=$ $25 \mathrm{~ms}$ [27]. In contrast to conventional time-of-flight expansion, where the initial spatial distribution of the sample influences the obtained momentum distribution-especially at low momenta-distortions are negligible in this method.

By combining the interaction quench with the projection onto molecules and the $\tau / 4$ momentum imaging, we are able to access the radial in situ pair momentum distribution $\tilde{n}(k)$ in the whole crossover regime.

Figure 2(b) shows the obtained pair momentum distributions for the coldest attainable temperature at different interaction strengths. One observes a dramatic enhancement at low momenta which manifests itself in a sharp central peak. This feature is strongest on the BEC side and persists above $\ln \left(k_{F} a_{2 D}\right)=0$ and the 3D Feshbach resonance, until it vanishes at $\ln \left(k_{F} a_{2 D}\right) \approx 3.2$ on the BCS side. Comparing the data at the two largest depicted values of $\ln \left(k_{F} a_{2 D}\right)$, one observes that the peak momentum density $\tilde{n}_{0}$ changes by almost an order of magnitude, whereas the in situ peak density $n_{0}$ changes by less than $10 \%$. As $\tilde{n}_{0}$ is a measure for the longrange coherence of the system [45], the observed abrupt change indicates the phase transition to the condensed phase.

For a more quantitative analysis of our data, we azimuthally average the pair momentum distribution. Figure 3(a) shows the obtained radial distribution for the coldest accessible temperature measured at $782 \mathrm{G}$, which corresponds to $\ell_{z} / a_{3 D}=1.55\left[\ln \left(k_{F} a_{2 D}\right) \approx-0.51\right]$. We extract the temperature $T$ of the sample from the high momentum tail of the radial distribution, which is well described by a Gaussian. Note that before the ramp of the interaction strength, the thermal part of the gas consists of molecules for $\ell_{z} / a_{3 D}>0.55$, free atoms for $\ell_{z} / a_{3 D}<-0.46$, and a mixture of atoms and molecules between these two interaction strengths [27]. For each investigated interaction strength and temperature, we determine the Fermi wave vector and the Fermi temperature from the in situ peak density according to $k_{F}^{2}=2 m k_{B} T_{F} / \hbar^{2}=4 \pi n_{0}$. Here, $m$ refers to the mass of a ${ }^{6} \mathrm{Li}$ atom and $k_{B}$ is Boltzmann's constant. This definition employs the local density approximation at the trap center and allows us to compare the obtained data to predictions for the homogeneous system. Note that $n_{0}=n_{0,|1\rangle}=n_{0,|2\rangle}$, where $n_{0,|i\rangle}$ is the peak density of atoms in state $|i\rangle$.

At low momenta, a fraction of the momentum density lies above the Gaussian fit [the gray area in Fig. 3(a)]. We define this quantity as the nonthermal fraction $N_{q} / N$ [46] and investigate its behavior as a function of the degeneracy temperature $T / T_{F}$ [see Fig. 3(b)]. While the non-Gaussian fraction vanishes for $T / T_{F} \gtrsim 0.5$, it slowly grows for
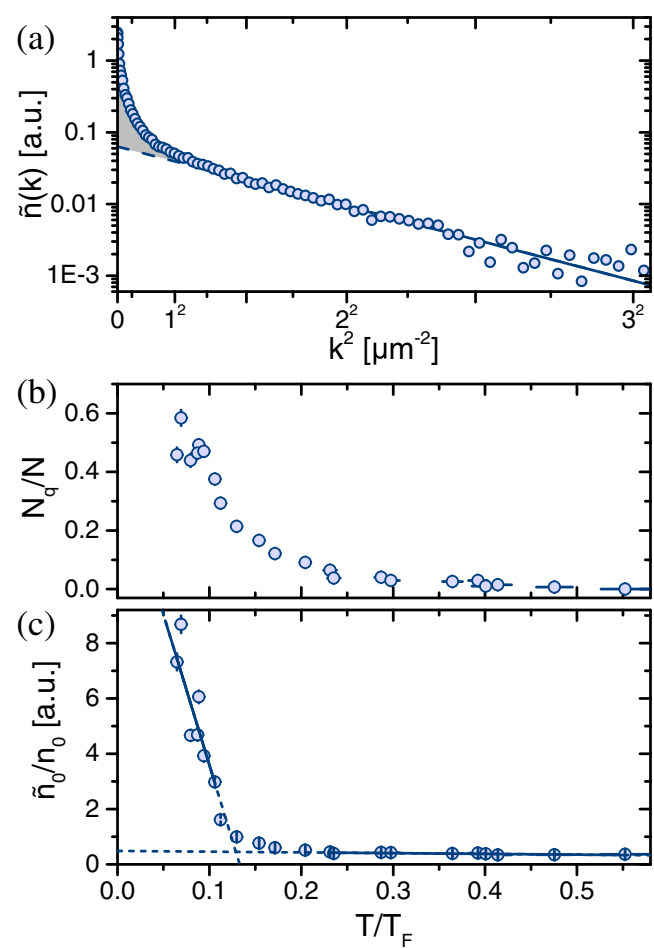

FIG. 3 (color online). Quantitative analysis of the momentum distribution at $\ell_{z} / a_{3 D}=1.55$. (a) Radial momentum distribution $\tilde{n}(k)$ at the coldest accessible temperature. We logarithmically plot $\tilde{n}(k)$ as a function of $k^{2}$. The thermal wing thus appears as a straight line from which we extract the temperature of the sample with a Boltzmann fit (line). The figure is the average of about 30 individual measurements. (b) Nonthermal fraction $N_{q} / N$ as a function of $T / T_{F} . N_{q}$ is indicated by the gray area in (a). (c) Normalized peak momentum density $\tilde{n}_{0} / n_{0}$ as a function of $T / T_{F}$. The intersection of linear fits to the high and low temperature regime yields the critical temperature $T_{c} / T_{F}$. Each data point in (b) and (c) is the average of about 30 individual measurements; the error bars indicate the standard error of the mean. Solid lines indicate the fitted data range.

decreasing temperatures. For $T / T_{F} \lesssim 0.2$, the slope increases until we reach $N_{q} / N \approx 0.6$ for the coldest samples. This is in agreement with theoretical predictions [47-49] and previous experimental results [40,45,50,51], which find a presuperfluid increase of low-momentum states for temperatures above the superfluid transition temperature $T_{c}$. This behavior inhibits a precise determination of the transition temperature $T_{c}$ from $N_{q} / N$. To obtain an estimate for the critical temperature, we instead plot the normalized peak momentum density $\tilde{n}_{0} / n_{0}$ as a function of temperature, as shown in Fig. 3(c). This quantity is a measure for the fraction of the sample which exhibits long-range phase coherence [45]. The innermost pixel of the momentum distribution corresponds to a coherence length well above $20 \mu \mathrm{m}$, which is approximately 1 order of magnitude larger than the thermal wavelength of the coldest samples. The normalized peak momentum density shows a sudden change of slope which we assume will occur at the phase transition. We estimate 


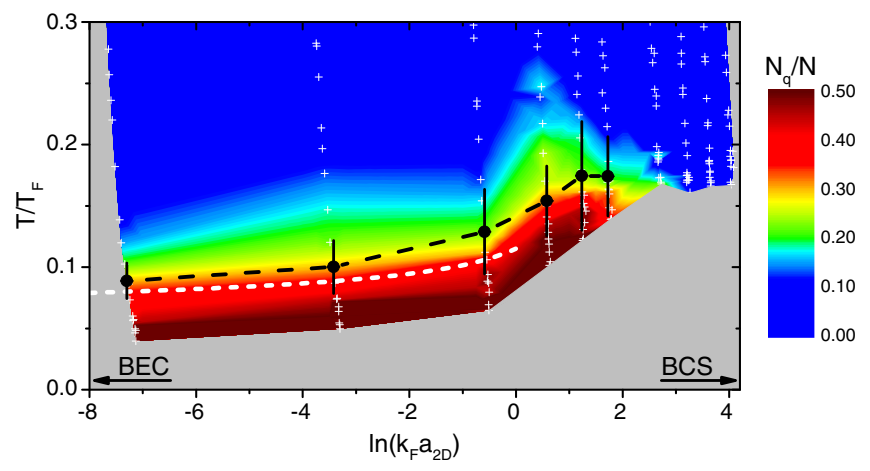

FIG. 4 (color online). Phase diagram of the strongly interacting 2D Fermi gas. The experimentally determined critical temperature $T_{c} / T_{F}$ is shown as black data points and the error bars indicate the statistical errors. Systematic uncertainties are discussed in detail in Ref. [27]. The color scale indicates the nonthermal fraction $N_{q} / N$ and is linearly interpolated between the measured data points (the white crosses). Each data point is the average of about 30 measurements. The dashed white line is the theoretical prediction for the BKT transition temperature given in Ref. [52].

$T_{c} / T_{F}$ by the intersection of linear fits to the regimes above and below the phase transition. For the example shown in Fig. 3, this results in a critical temperature of $T_{c} / T_{F}=0.129(35)$, where the statistical uncertainty is obtained from the standard errors of the two linear fits. The critical phase space density is $\rho_{c}=n_{0, c} \lambda_{d B, c}^{2}=3.9(6)$, where $\lambda_{d B, c}$ and $n_{0, c}$ are the thermal de Broglie wavelength and the peak in situ density at the critical temperature, respectively.

By repeating this analysis for all investigated interaction strengths, we obtain the transition temperature as a function of the interaction parameter $\ln \left(k_{F} a_{2 D}\right)$. The resulting values are shown as black dots in Fig. 4 together with the corresponding non-Gaussian fraction $N_{q} / N$, which is displayed as a color scale. Comparing the data for $T_{c} / T_{F}$ and $N_{q} / N$, one finds that the phase transition occurs at a significant non-Gaussian fraction of $N_{q} / N \approx$ 0.3 for all measured interaction strengths.

On the BEC side of the phase diagram, one observes a slow increase of the measured critical temperature towards the crossover region. Within their statistical uncertainties, the measured values of $T_{c} / T_{F}$ are in good agreement with an effective description in terms of 2D bosons [52]. This theoretical prediction describes a BKT transition into a superfluid phase with algebraically decaying phase coherence. Interestingly, the bosonic theory provides a reasonable description of the data up to $\ln \left(k_{F} a_{2 D}\right)=0$, where the 2D scattering amplitude diverges. This indicates that the fermionic nature of the constituents of the bosonic dimers has only a small effect on the many-body physics of the system up to this point. The crossover to a fermionic description should thus occur at positive values of $\ln \left(k_{F} a_{2 D}\right)$. This is in line with recent theoretical predictions $[6,53]$.

Far on the BCS side, fermionic theories predict an exponential decrease of $T_{c} / T_{F}$ [7,54]. Although we can only give an upper limit for the critical temperature $T_{c} / T_{F} \leq 0.16$ for $\ln \left(k_{F} a_{2 D}\right) \geq 2$, the observed non-Gaussian fraction is consistent with a decrease towards the BCS limit. However, $T_{c} / T_{F}$ is systematically above the theoretical predictions for $\ln \left(k_{F} a_{2 D}\right)>0$ [7,8,52]. Part of this deviation might be due to the residual influence of the third dimension. In our system, residual axial excitations grow with increasing $\ln \left(k_{F} a_{2 D}\right)$ [27]. Recently, it was predicted that they would lead to an increased critical temperature [55]. Additionally, the threedimensional internal structure of atom pairs might lead to corrections in the regime where $E_{B} \approx \hbar \omega_{z}$, which go beyond the two-body sector. Whether this effect has any influence on the measured phase diagram still needs further experimental and theoretical consideration. Initial steps in this direction have been taken [56].

Our work constitutes a basis for future theoretical and experimental studies of quantum gases in the quasi-2D BEC-BCS crossover. The measured critical temperature suggests the validity of BKT theory on the bosonic side. Superfluidity and the algebraic decay of correlations below the transition remain to be validated. Indeed, our ability to extract the in situ momentum distribution with negligible distortion offers direct access to the coherence properties of the system. A first analysis of the trap averaged first order correlation function, which we obtain by Fourier transforming the pair momentum distribution, suggests algebraically decaying phase correlations below the critical temperature. However, because of the inhomogeneity of our system, a careful analysis is required to unambiguously confirm the BKT nature of the observed transition. Additionally, the equation of state can be extracted from the density distribution in the trap. Finally, the exploration of the dimensional crossover to $3 \mathrm{D}$, in which an increased $T_{c} / T_{F}$ is predicted [55], offers new opportunities to understand mechanisms which lead to high critical temperatures.

The authors would like to thank T. Enss, J. Levinsen, P. Massignan, L. Mathey, M. M. Parish, and J. M. Pawlowski for the valuable discussions, and J. H. Becher, J. E. Bohn, and S. Pres for their contributions to the construction of the 2D setup. The authors also gratefully acknowledge support from ERC Starting Grant No. 279697, ERC Advanced Grant No. 290623, the Helmholtz Alliance HA216/EMMI, and the Heidelberg Center for Quantum Dynamics. M. G. R. and I. B. acknowledge support by the Landesgraduiertenförderung Baden-Württemberg.

M. G. R., A.N.W., and G.Z. contributed equally to this work.

*To whom all correspondence should be addressed. mries@physi.uni-heidelberg.de

Present address: MIT-Harvard Center for Ultracold Atoms, MIT, Cambridge, MA 02139, USA.

[1] M. R. Norman, Science 332, 196 (2011).

[2] P. A. Lee, N. Nagaosa, and X.-G. Wen, Rev. Mod. Phys. 78, 17 (2006). 
[3] J. Levinsen and M. M. Parish, Annu. Rev. Cold At. Mol. 3, 1 (2015).

[4] M. Randeria, J.-M. Duan, and L.-Y. Shieh, Phys. Rev. Lett. 62, 981 (1989).

[5] M. Iskin and C. A. R. Sá de Melo, Phys. Rev. Lett. 103, 165301 (2009).

[6] G. Bertaina and S. Giorgini, Phys. Rev. Lett. 106, 110403 (2011).

[7] M. Bauer, M. M. Parish, and T. Enss, Phys. Rev. Lett. 112, 135302 (2014).

[8] M. Matsumoto and Y. Ohashi, J. Phys. Conf. Ser. 568, 012012 (2014).

[9] I. Bloch, J. Dalibard, and W. Zwerger, Rev. Mod. Phys. 80, 885 (2008).

[10] Z. Hadzibabic, P. Krüger, M. Cheneau, B. Battelier, and J. Dalibard, Nature (London) 441, 1118 (2006).

[11] R. Desbuquois, L. Chomaz, T. Yefsah, J. Leonard, J. Beugnon, C. Weitenberg, and J. Dalibard, Nat. Phys. 8, 645 (2012).

[12] M. Bartenstein, A. Altmeyer, S. Riedl, S. Jochim, C. Chin, J. H. Denschlag, and R. Grimm, Phys. Rev. Lett. 92, 203201 (2004).

[13] C. A. Regal, M. Greiner, and D. S. Jin, Phys. Rev. Lett. 92 , 040403 (2004).

[14] M. W. Zwierlein, C. A. Stan, C. H. Schunck, S. M. F. Raupach, A. J. Kerman, and W. Ketterle, Phys. Rev. Lett. 92, 120403 (2004).

[15] T. Bourdel, L. Khaykovich, J. Cubizolles, J. Zhang, F. Chevy, M. Teichmann, L. Tarruell, S. J. J. M. F. Kokkelmans, and C. Salomon, Phys. Rev. Lett. 93, 050401 (2004).

[16] K. Martiyanov, V. Makhalov, and A. Turlapov, Phys. Rev. Lett. 105, 030404 (2010).

[17] M. Feld, B. Fröhlich, E. Vogt, M. Koschorreck, and M. Köhl, Nature (London) 480, 75 (2011).

[18] P. Dyke, E. D. Kuhnle, S. Whitlock, H. Hu, M. Mark, S. Hoinka, M. Lingham, P. Hannaford, and C. J. Vale, Phys. Rev. Lett. 106, 105304 (2011).

[19] M. Koschorreck, D. Pertot, E. Vogt, B. Fröhlich, M. Feld, and M. Köhl, Nature (London) 485, 619 (2012).

[20] A. T. Sommer, L. W. Cheuk, M. J. H. Ku, W. S. Bakr, and M. W. Zwierlein, Phys. Rev. Lett. 108, 045302 (2012).

[21] V. Makhalov, K. Martiyanov, and A. Turlapov, Phys. Rev. Lett. 112, 045301 (2014).

[22] N. D. Mermin and H. Wagner, Phys. Rev. Lett. 17, 1133 (1966).

[23] P. C. Hohenberg, Phys. Rev. 158, 383 (1967).

[24] V. L. Berezinskii, Sov. Phys. JETP 34, 610 (1972).

[25] J. M. Kosterlitz and D. J. Thouless, J. Phys. C 6, 1181 (1973).

[26] G. Zürn, T. Lompe, A. N. Wenz, S. Jochim, P. S. Julienne, and J. M. Hutson, Phys. Rev. Lett. 110, 135301 (2013).

[27] See Supplemental Material at http://link.aps.org/supplemental/ 10.1103/PhysRevLett.114.230401 for details on the experimental setup, the experimental methods, and the data evaluation, which includes Refs. [28-35].

[28] D. S. Petrov and G. V. Shlyapnikov, Phys. Rev. A 64, 012706 (2001).

[29] D. S. Petrov, C. Salomon, and G. V. Shlyapnikov, Phys. Rev. A 71, 012708 (2005).

[30] B. Fröhlich, M. Feld, E. Vogt, M. Koschorreck, W. Zwerger, and M. Köhl, Phys. Rev. Lett. 106, 105301 (2011).

[31] G. Reinaudi, T. Lahaye, Z. Wang, and D. Guéry-Odelin, Opt. Lett. 32, 3143 (2007).

[32] T. Yefsah, R. Desbuquois, L. Chomaz, K. J. Günter, and J. Dalibard, Phys. Rev. Lett. 107, 130401 (2011).
[33] M. E. Gehm, Properties of ${ }^{6} \mathrm{Li}$, http://www.physics.ncsu .edu/jet/techdocs/pdf/PropertiesOfLi.pdf.

[34] C. F. Ockeloen, A. F. Tauschinsky, R. J. C. Spreeuw, and S. Whitlock, Phys. Rev. A 82, 061606 (2010).

[35] W. Ketterle and M. W. Zwierlein, Riv. Nuovo Cimento Soc. Ital. Fis. 31, 247 (2008).

[36] For $E_{B} \gg \hbar \omega_{z}$, the system consists of tightly bound bosonic molecules whose internal structure is not resolved. For $E_{B} \ll$ $\hbar \omega_{z}$ the internal structure of the dimers is well described within the 2D framework. Only for $E_{B} \approx \hbar \omega_{z}$, the internal structure is influenced by the third spatial dimension and can deviate from the $2 \mathrm{D}$ predictions. This affects the short-range behavior of the system. However, we expect the influence on the long-range behavior to be negligible (see Sec. IV in Ref. [27]).

[37] D. S. Petrov, M. Holzmann, and G. V. Shlyapnikov, Phys. Rev. Lett. 84, 2551 (2000).

[38] I. Shvarchuck, C. Buggle, D. S. Petrov, K. Dieckmann, M. Zielonkowski, M. Kemmann, T. G. Tiecke, W. von Klitzing, G. V. Shlyapnikov, and J. T. M. Walraven, Phys. Rev. Lett. 89, 270404 (2002).

[39] A. H. van Amerongen, J. J. P. van Es, P. Wicke, K. V. Kheruntsyan, and N. J. van Druten, Phys. Rev. Lett. 100, 090402 (2008).

[40] S. Tung, G. Lamporesi, D. Lobser, L. Xia, and E. A. Cornell, Phys. Rev. Lett. 105, 230408 (2010).

[41] P. A. Murthy, D. Kedar, T. Lompe, M. Neidig, M. G. Ries, A. N. Wenz, G. Zürn, and S. Jochim, Phys. Rev. A 90, 043611 (2014).

[42] Because of this projection, information about the relative momentum of the paired atoms is lost. We therefore do not observe the Tan contact in the pair momentum distribution [43].

[43] S. Tan, Ann. Phys. (Amsterdam) 323, 2971 (2008).

[44] M. W. Zwierlein, J. R. Abo Shaeer, A. Schirotzek, C. H. Schunck, and W. Ketterle, Nature (London) 435, 1047 (2005).

[45] T. Plisson, B. Allard, M. Holzmann, G. Salomon, A. Aspect, P. Bouyer, and T. Bourdel, Phys. Rev. A 84, 061606 (2011).

[46] We identify the non-Gaussian fraction $N_{q} / N$ with the fraction of the cloud which has non-Gaussian fluctuations. In the literature $[47,48]$, this is referred to as the quasicondensate. Below $T_{c}$, the quasicondensate density becomes identical to the superfluid density in mean-field theory.

[47] N. Prokof'ev, O. Ruebenacker, and B. Svistunov, Phys. Rev. Lett. 87, 270402 (2001).

[48] N. Prokof'ev and B. Svistunov, Phys. Rev. A 66, 043608 (2002).

[49] R. N. Bisset, M. J. Davis, T. P. Simula, and P. B. Blakie, Phys. Rev. A 79, 033626 (2009).

[50] P. Cladé, C. Ryu, A. Ramanathan, K. Helmerson, and W. D. Phillips, Phys. Rev. Lett. 102, 170401 (2009).

[51] C.-L. Hung, X. Zhang, N. Gemelke, and C. Chin, Nature (London) 470, 236 (2011).

[52] D. S. Petrov, M. A. Baranov, and G. V. Shlyapnikov, Phys. Rev. A 67, 031601 (2003).

[53] V. Ngampruetikorn, J. Levinsen, and M. M. Parish, Phys. Rev. Lett. 111, 265301 (2013).

[54] C. A. R. Sá de Melo, M. Randeria, and J. R. Engelbrecht, Phys. Rev. Lett. 71, 3202 (1993).

[55] A. M. Fischer and M. M. Parish, Phys. Rev. B 90, 214503 (2014).

[56] P. Dyke, K. Fenech, T. Peppler, M. G. Lingham, S. Hoinka, W. Zhang, B. Mulkerin, H. Hu, X. J. Liu, and C. J. Vale, arXiv:1411.4703. 